\title{
Modelo de indagación y las habilidades para argumentar: resolver problemas en ciencias con estudiantes de grado Once del Colegio Enrique Olaya Herrera
}

\section{Model of inquiry and the skills to argue: solve problems in science with eleventh grade students at Enrique Olaya Herrera School}

\author{
Heidy Fonseca ${ }^{1}$ \\ Paula Muñoz ${ }^{2}$ \\ Cindy Palacios ${ }^{3}$ \\ Lorena Torres ${ }^{4}$ \\ Quira Sanabria $^{5}$
}

\section{Resumen}

El siguiente documento expone los resultados obtenidos en la práctica pedagógica realizada en la clase de Química del Colegio Enrique Olaya Herrera de Bogotá, que buscó promover las habilidades para argumentar cuando se construyen explicaciones sobre alguna situación problema, a partir del uso del modelo de indagación, el cual es evaluado bajo la taxonomía. Como resultado general se destaca que los estudiantes reflejan un aprendizaje multi-estructural, es decir que la población asume la existencia del sistema teórico y reconoce términos como conceptos. Sin embargo, difícilmente relacionan el lenguaje científico con las situaciones problemas sugeridas como análisis.

Palabras claves

Indagación, argumentación, taxonomía soLo, Química.

1 Estudiante de Licenciatura en Química de la Universidad Pedagógica Nacional. Correo electrónico: dqu_hcfonsecas272@pedagogica.edu.co.

2 Estudiante de Licenciatura en Química de la Universidad Pedagógica Nacional. Correo electrónico: dqu_pmunoz072@pedagogica.edu.co.

3 Estudiante de Licenciatura en Química de la Universidad Pedagógica Nacional. Correo electrónico: dqu_cpalacios239@pedagogica.edu.co.

4 dqu_lltorresm19o@pedagogica.edu.co.

5 Asesora de práctica de la Universidad Pedagógica Nacional. Correo electrónico: qsanabria@pedagogica.edu.co 


\section{Abstract}

The following document presents the results obtained in the pedagogical practice carried out in the chemistry class of the Enrique Olaya Herrera School in Bogotá, focused on promoting the skills to argue when explanations are constructed about a problem situation, based on the use of the model of inquiry, which is evaluated under the SOLO taxonomy.

As a general result it stands out that the students reflect a multi-structural learning, that is to say that the population assumes the existence of the theoretical system, recognizes terms as concepts, however, they hardly relate the scientific language with the situations suggested as analysis problems.

\section{Keywords}

Inquiry, argumentation, taxonomy soLo, chemistry.

\section{Introducción}

La Institución Educativa Distrital Enrique Olaya Herrera, ubicado en la carrera $10 .^{a}{ }^{n} \cdot{ }^{\circ} 31-29$ sur, en la localidad 18 de Rafael Uribe Uribe, está desarroIlando actualmente un proyecto de pensamiento lógico, el cual, entre sus principales objetivos, quiere ejercitar y estimular en sus estudiantes las habilidades de investigar, comunicar y argumentar para resolver problemas.

Teniendo en cuenta el proyecto institucional que se está desarrollando en el colegio, las docentes en formación en el área de Química (L.F) de la Universidad Pedagógica Nacional pretenden reforzar el desarrollo del proyecto institucional mediante una secuencia de actividades basadas en el modelo de indagación científica, con el cual los estudiantes de grado Once de la institución analicen, argumenten, razonen, justifiquen y prueben sus razonamientos para la solución de problemas.

De acuerdo con lo anterior, el proyecto propone y desarrolla una secuencia de actividades a partir del modelo de indagación científica, que se refiere a las diversas formas por las cuales los científicos abordan el conocimiento de la naturaleza y proponen explicaciones basadas en las pruebas derivadas de su trabajo. La indagación, como lo indica Schwab también se refiere a las actividades estudiantiles en las cuales se desarrollan conocimiento y entendimiento de las ideas científicas; por ejemplo, como aquellas que surgen en el desarrollo de experiencias en el laboratorio.

Para reforzar el proyecto institucional, se escogió el modelo de indagación porque permite articular un conjunto de actividades en las que implica hacer observaciones, plantear preguntas, examinar libros y otras fuentes de información para ver lo que es ya conocido, proponer respuestas, dar explicaciones y comunicar los resultados (Garritz, 2010).

Teniendo en cuenta lo anterior, se planteó la siguiente pregunta problema: ¿Cómo el modelo de indagación promueve las habilidades para argumentar y resolver problemas en ciencias en estudiantes de grado Once del Colegio Enrique Olaya Herrera?

\section{Marco de referencia}

La inclusión de la indagación en el currículo de ciencias de la primaria y la secundaria fue recomendada por John Dewey; él insistió en que los profesores utilizaran la indagación como una estrategia de enseñanza, aprovechando los seis pasos conocidos en la formación del conocimiento: a) detectar situaciones desconcertantes; b) aclarar el problema; c) formular una hipótesis tentativa; d) probar dicha hipótesis; e) revisarla a través de pruebas rigurosas, y f) actuar sobre la solución. En el modelo de Dewey, el estudiante es participativo y está involucrado activamente, mientras que el profesor es un guía y un facilitador (Garritz, 2010).

En la búsqueda de las capacidades que promueve la indagación, se escogieron los criterios especificados en nrc (1996) para el nivel de bachillerato:

1. Identificar preguntas y conceptos que guíen las investigaciones (los estudiantes formulan una hipótesis probable y un diseño experimental apropiado para ser utilizado). 
2. Diseñar y conducir investigaciones científicas (con el empleo de conceptos claros y bien definidos, el equipo apropiado, precauciones de seguridad, empleo de tecnologías, etc., los estudiantes deben buscar pruebas, aplicar la lógica, poner a prueba sus hipótesis y construir un argumento para las explicaciones propuestas).

3. Utilizar las tecnologías más apropiadas y la matemática para mejorar las investigaciones y su comunicación.

4. Formular y revisar las explicaciones y modelos científicos mediante el empleo de la lógica y las pruebas científicas (la indagación estudiantil debiera resultar en una explicación o un modelo plausible o científico).

5. Reconocer y analizar explicaciones y modelos alternativos (revisar el entendimiento científico actual y reunir pruebas para determinar cuáles explicaciones del modelo son las mejores).

6. Comunicar y defender un argumento científico (los estudiantes deben refinar sus habilidades y reunir presentaciones orales y por escrito que involucren las respuestas a los comentarios críticos de sus pares).

Estos criterios fueron complementarios con los criterios de evaluación a partir de la teoría del alineamiento constructivo, planteada por Biggs (1982), que acude a los constructos sobre los enfoques de enseñanza y de aprendizaje y que incluyen el modelo 3P y la taxonomía solo (Structure of Observed Learning Outcomes) (Soler, 2015).

El modelo 3P ve el proceso de enseñanza y de aprendizaje como un ecosistema en el que todas las partes que lo conforman son solidarias entre sí, de modo que, lo que ocurre en una parte del sistema, inevitablemente afecta a las demás partes, y por supuesto al sistema en general.

La taxonomía solo es un mecanismo sistemático para describir cómo se incrementa la complejidad de la actuación de un estudiante al enfrentarse a las tareas académicas. Puede ser utilizada, entonces, para plantear los objetivos curriculares, que indiquen dónde deben estar operando los estudiantes, también para evaluar los resultados del aprendizaje, así se puede saber en qué nivel concreto se están desenvolviendo. Como se verá más adelante, en la figura 1 se plantean los niveles de compresión que pueden adquirir los estudiantes acerca de la temática molaridad al terminar la actividad propuesta, según la taxonomía solo y explicada por Biggs.

\section{Objetivo general}

- Evaluar las habilidades para argumentar y resolver problemas en ciencias en los estudiantes de grado Once del Colegio Enrique Olaya Herrera, a través del modelo de indagación.

\section{Objetivos específicos}

- Establecer las categorías de análisis de las habilidades para argumentar y resolver problemas en ciencias de acuerdo con los criterios del alineamiento constructivo.

- Diseñar e implementar una ruta de aprendizaje a través del modelo de indagación.

\section{Metodología}

Para este proceso metodológico se acogió una perspectiva cualitativa, a partir del uso de instrumentos ya diseñados dentro de la metodología propuesta. La población está compuesta por los estudiantes de grado Décimo y Once de la jornada mañana del Colegio Enrique Olaya Herrera. En correspondencia con el objetivo planteado en la propuesta para desarrollar la práctica pedagógica, se eligió como muestra a los estudiantes de grado Once, de tres cursos los cuales culminaron todas las actividades planteadas. 
A partir de diseños instrumntales se evidenciarán las ideas previas que los esudantes poseen para abarcar las temáticas a partir de modelo de indagación.

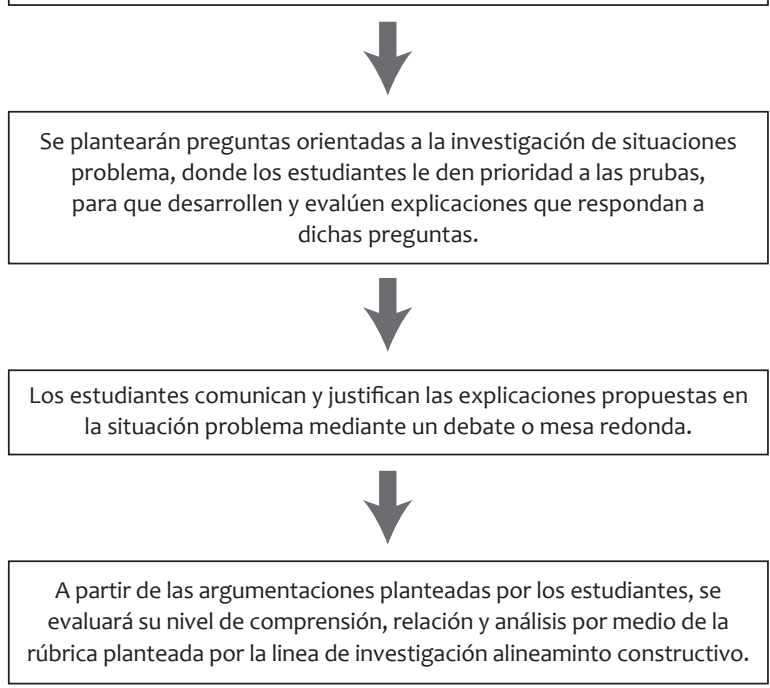

Figura 1. Representación del proceso metodológico

Fuente: elaboración propia.

\section{Resultados}

La primera actividad realizada fue una prueba diagnóstica (ver anexo $\mathrm{A}$ ) de ideas previas que tienen los estudiantes de grado Once con respecto a masa, peso, volumen, densidad, solubilidad y unidades de concentración de soluciones.
Se aplicó la prueba de ideas previas a estudiantes del curso 11-01, 11-02 y 11-04 sobre soluciones químicas, la cual se resolvió en dos sesiones de clase, a saber:

- En un primer momento, se entregó el instrumento que constaba de dos partes. En la primera cada estudiante debía responder a las preguntas de manera individual y en la segunda parte los estudiantes debían realizar grupos conformados por cinco estudiantes para resolver los ejercicios, con el fin de aplicar las ecuaciones de concentraciones químicas.

- En un segundo momento, se llevó a cabo una práctica experimental sobre las soluciones químicas, el cual se volvió propósito de reflexión para la construcción de argumentos por medio de una uve $(v)$ heurística en la cual se consignaron los resultados de la práctica de laboratorio, y que se convirtió en el producto para ser analizado en cuanto a el uso de la información disponible, el problema planteado y la forma como los estudiantes construyeron una explicación válida según sus propias comprensiones.

Los resultados obtenidos se analizaron bajo una rúbrica de evaluación teniendo en cuenta la taxonomía solo y el modelo 3P.

De acuerdo con esto, se presentan los siguientes resultados:
Preguntas con respuesta abierta

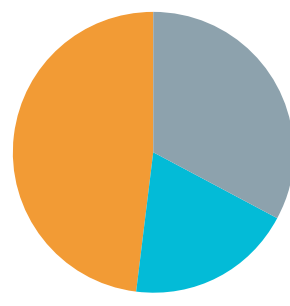

Preguntas de análisis y resolución de problemas

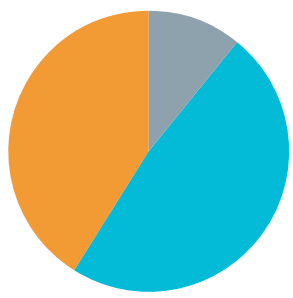

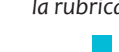

1
2

3

4
Categorías de la rubrica

ategorías de la rubrica
Preguntas deinterpretación y aplicación de...

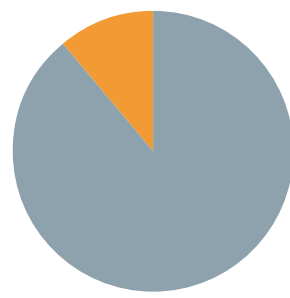

Categorías de la rubrica

Figura 2. Resultados prueba diagnóstica curso 11-04.

Fuente: elaboración propia. 


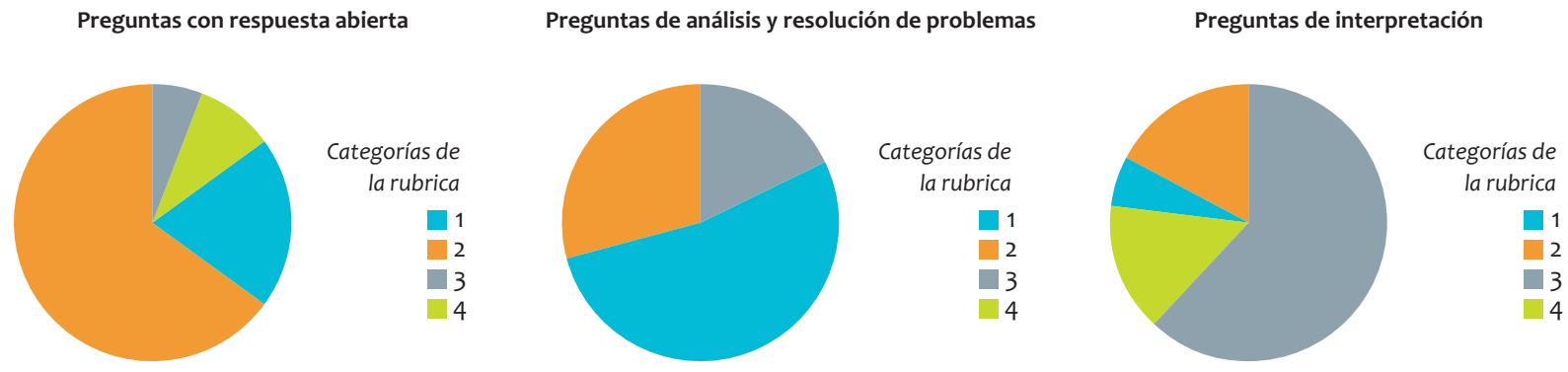

Figura 3. Resultados prueba diagnóstica curso 11-02.

Fuente: elaboración propia.

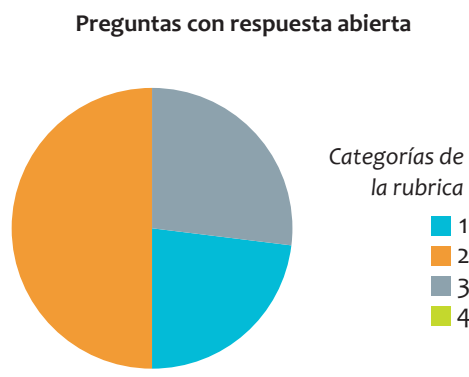

Preguntas de análisis y resolución de problemas

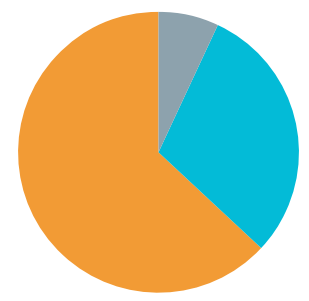

Categorías de la rubrica

1
$\square$
$-\quad 3$
-4
4

Preguntas de interpretación

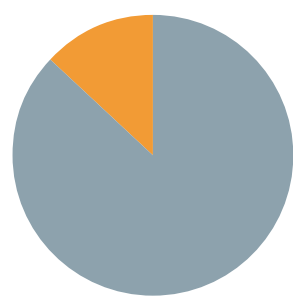

Categorías de la rubrica

1

2

4

Figura 4. Resultados prueba diagnóstica curso 11-01.

Fuente: elaboración propia.

En la segunda actividad, se propuso un segundo instrumento, que se diseñó para realizar una segunda práctica de laboratorio sobre el comportamiento de las soluciones químicas; en este caso participaron 34 estudiantes del curso 1104 y se dividió en tres sesiones, en las que se sugirió una ruta distinta para los momentos de reflexión teórica:

- Primera sesión: explicación anticipada de la actividad.

- Segunda sesión: desarrollo de la práctica de laboratorio
- Tercera sesión: socialización y presentación de informe.

La recolección de resultados, en este caso, se hizo a partir de una uve (v) heurística que fue elaborada de manera individual. Con el fin de contrastar con los resultados que ya se tenían de los grupos de trabajo, el análisis se hizo utilizando la misma rúbrica diseñada en el momento anterior. Las figuras que siguen a continuación representan los resultados obtenidos. 


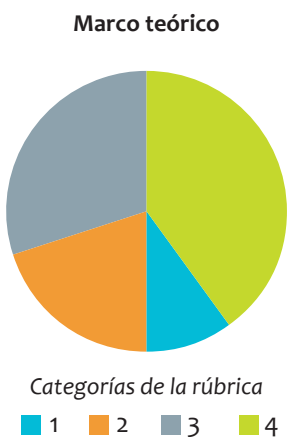

\begin{tabular}{l|l|l|l}
1 & 2 & 3 & 4
\end{tabular}

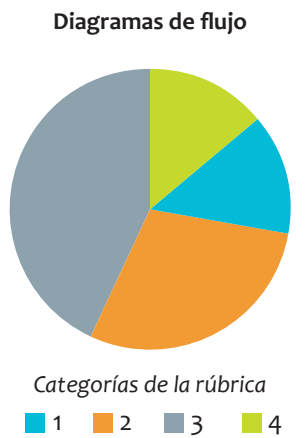

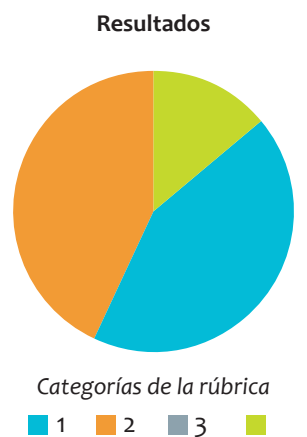

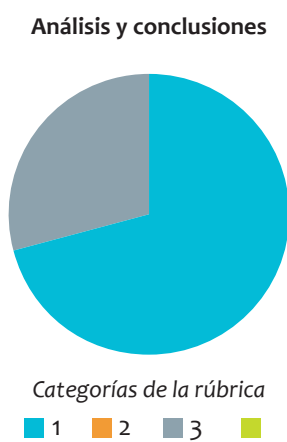

Figura 5. Resultados de práctica de laboratorio aplicada a 11-04

Fuente: elaboración propia.

\section{A modo de conclusión}

A partir de la implementación de los dos instrumentos diseñados con respecto al modelo de indagación y el alineamiento constructivo asumidos como marco de referencia, en los diferentes cursos de grado Once, posterior a plantear por lo menos dos estrategias diferentes de abordaje de las mismas temáticas, se puede afirmar que los estudiantes aprenden a través de distintas actividades en las cuales son ellos quienes ejecutan acciones propias para documentarse, y luego, construir argumentos frente a un problema planteado. Lo anterior sugiere, dentro de la taxonomía solo, que es válido afirmar que las personas van profundizando sus niveles de comprensión de forma paulatina. Comprender y debatir en grupos de pares facilitó que los estudiantes mejoraran en una segunda oportunidad la elaboración de argumentos. Esta evidencia permite afirmar que se avanza desde un nivel de conocimiento declarativo y procedimental hacia un nivel de conocimiento condicional y funcional.

Los análisis hechos sobre estas construcciones escritas permitió al equipo de trabajo obtener información relevante sobre los niveles de aprendizaje de los estudiantes del Colegio Enrique Olaya Herrera, de modo que se categorizaron en función del marco de referencia como un grupo que mayoritariamente presentaron un aprendizaje multi-estructural. Esta clasificación sugirió que los estudiantes tienen conocimiento de los conceptos planteados, puesto que los asumen como existentes; sin embargo, no logran relacionarlos dentro de un sistema teórico propio, lo cual se vio reflejado en los argumentos construidos según el uso que le dieron al lenguaje científico en la explicación de las situaciones presentadas.

Estos resultados sugieren que evaluar las habilidades de pensamiento implica revisar las estrategias que se proponen para el trabajo en el aula, así como considerar las implicaciones que tiene en el trabajo colectivo e individual las actitudes de los estudiantes frente al aprendizaje de la química en este caso. Así, se pueden logar escenarios más favorables que promuevan alternativas de solución frente a dificultades conceptuales ya identificadas en los ejercicios de diagnóstico, por lo cual se afirma que el modelo de indagación es una estrategia didáctica apropiada para diseñar condiciones más adecuadas al considerar las metas de aprendizaje como objetivos alcanzables, a partir de promover la autoregulación tal como se sugiere desde la estructura del modelo $3 \mathrm{p}$.

\section{Referencias}

Biggs, J. B., y Collis, K. F. (1982). Evaluating the quality of learning: The taxonomy. Nueva York: Academic Press.

Garritz, A. (2010). Indagación: las habilidades para desarrollarla y promover el aprendizaje. Educación quimica, 21(2), 106-110. 
National Research Council, NRC. (1996). National Science Education Standards. Academic Press, 23.
Schwab, J. (1960-1966-1978). The teaching of science. Cambridge Harvard University Press, 6-11.

\title{
Anexo A. Prueba diagnóstica
}

\author{
Universidad Pedagógica Nacional de Colombia \\ Facultad de Ciencia y Tecnología \\ Departamento de Química \\ Colegio Enrique Olaya Herrera \\ Prueba diagnóstica
}

\section{Disoluciones}

\section{OBJETIVOS:}

Caracterizar los conceptos previos que poseen los alumnos sobre el comportamiento de las disoluciones.

Diseñar metodologías que favorezcan el aprendizaje sobre el comportamiento de las disoluciones a través del modelo pedagógico indagación en correspondencia con la perspectiva del alineamiento constructivo.

\section{Preguntas abiertas}

Objetivo: Identificar a partir de preguntas abiertas qué ideas previas poseen los estudiantes acerca de las definiciones que nos permiten empezar a abarcar la temática de soluciones.

Argumente con sus palabras las siguientes preguntas:

1. Qué entiende usted por:
A. Masa:
B. Peso:
C. Volumen:
D. Densidad:

2. Elabore un párrafo que articule las explicaciones anteriormente descritas en el listado de palabras.

\section{Objetivo: Identificar si los estudiantes pueden analizar y dar una solución a la situación \\ planteada a partir de sus conocimientos previos.}

3. Observe detenidamente la siguiente imagen, y de acuerdo con ella responda:

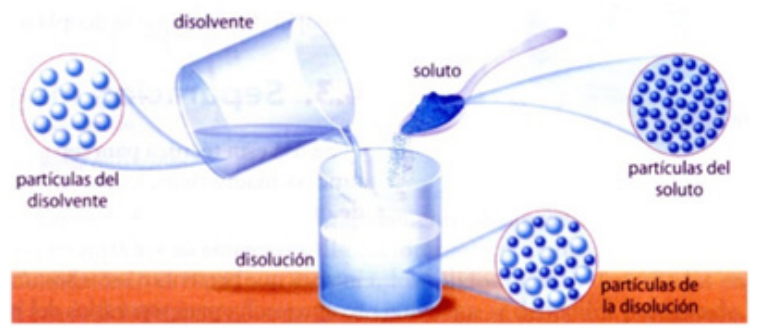

Imagen 1. Mezclas y sustancias puras.

Fuente: https://es.slideshare.net/tango67/ mezclas-y-sustancias-puras-3378039

- Caracterice los tipos de mezcla que usted conoce

- Elabore una explicación del fenómeno de solubilidad.

- Elabore una explicación para soluto y solvente. 


\section{Resolución de problemas}

\section{Objetivo: Identificar si los estudiantes \\ interpretan adecuadamente las ecuaciones para darle resolución a los problemas planteados.}

Resuelva los problemas que se presentan a continuación teniendo en cuenta la información de la tabla

\begin{tabular}{|c|c|}
\hline Concentración & Fórmula \\
\hline$\% \mathrm{~m} / \mathrm{m}$ (masa- masa) & $\begin{array}{c}\% \mathrm{~mm}=\text { masa solutomasa sol- } \\
\text { vente*100 }\end{array}$ \\
\hline$\% \mathrm{v} / \mathrm{v}$ (volumen-volumen) & $\begin{array}{c}\% \mathrm{vv}=\text { volumen soluto } \\
\text { volumen solvente*100 }\end{array}$ \\
\hline$\% \mathrm{~m} / \mathrm{v}$ (masa-volumen & $\begin{array}{c}\% \mathrm{mv}=\text { masa soluto } \\
\text { volumen solvente*100 }\end{array}$ \\
\hline
\end{tabular}

A. Una solución de azúcar en agua contiene $20 g$ de azúcar en $70 g$ de solvente. Expresar la solución en \%m/m.

B. Una solución salina contiene $30 g$ de cloruro de sodio en $80 \mathrm{~mL}$ de solución. Calcular su concentración en \%m/v.

C. Calcular la concentración en volumen de una solución alcohólica, que contiene $15 \mathrm{~mL}$ de alcohol disueltos en $65 \mathrm{~mL}$ de solución. 